\title{
Potentiation and overshadowing between landmarks and environmental geometric cues
}

\author{
Murray R. Horne • John M. Pearce
}

Published online: 21 April 2011

(C) Psychonomic Society, Inc. 2011

\begin{abstract}
Rats were required in three experiments to find one of two submerged platforms that were situated in the same pair of diagonally opposite corners of a rectangular grey swimming pool. The experimental groups were trained with landmarks, comprising A4 cards attached to the walls, located in the corners containing the platforms. For the control groups, the landmarks were situated in the corners containing the platforms for half of the trials, and in the other corners for the remaining trials. Learning about the positions of the platforms with reference to the shape of the pool was overshadowed in the experimental groups when the landmarks were white, and enhanced when the landmarks were black. A fourth experiment assessed whether geometric cues influenced the control acquired by the landmarks. As in the previous experiments, the presence of the geometric cues overshadowed learning about the landmarks when they were white, but enhanced learning when the landmarks were black.
\end{abstract}

Keywords Overshadowing · Potentiation · Landmarks · Geometry

There is abundant evidence showing that animals can locate a hidden goal by reference to the shape of their environment. Cheng (1986) provided the first demonstration of this effect when he required rats to find food that was hidden in one corner of a rectangular arena. Even in the absence of

M. R. Horne $(\varangle) \cdot$ J. M. Pearce

School of Psychology, Cardiff University,

70 Park Place,

Cardiff CF10 3AT Wales, UK

e-mail: HorneMR@cardiff.ac.uk

J. M. Pearce

e-mail: pearcejm@cardiff.ac.uk any other cues, searching for food was confined predominantly to the two geometrically correct corners. Subsequent studies have shown that animals can find a hidden goal successfully in an arena in the shape of a kite (Pearce, Good, Jones, \& McGregor, 2004), a parallelogram (Tommasi \& Polli, 2004), a triangle with a curved base (Pearce, Ward-Robinson, Good, Fussell, \& Aydin, 2001), and even a complex shape that can best be described as the outline of a house (McGregor, Jones, Good \& Pearce, 2006). In addition to rats, this ability to navigate with reference to what have been called geometric cues has been shown in monkeys (see, e.g., Gouteux, ThinusBlanc, \& Vauclair, 2001), pigeons (e.g., Bingman, Erichsen, Anderson, Good, \& Pearce, 2006), goldfish (e.g., Sovrano, Bisazza, \& Vallortigara, 2003), ants (Wystrach \& Beugnon, 2009), infant humans (e.g., Wang, Hermer, \& Spelke, 1999), and adult humans (e.g., Hermer \& Spelke, 1996).

In many of the experiments above, there were one or more landmarks located within the arena. The purpose of this manipulation was to determine whether the landmark would influence learning based on the shape of the environment. Unfortunately, the results from these experiments do not point to a clear-cut conclusion. In the majority of studies, no effect of the landmark was found (Hayward, Good, \& Pearce, 2004; Hayward, McGregor, Good, \& Pearce, 2003; McGregor, Horne, Esber, \& Pearce, 2009; Pearce et al., 2001; Wall, Botly, Black, \& Shettleworth, 2004); in others, the landmark disrupted learning based on the shape of the environment (Gray, Bloomfield, Ferrey, Spetch, \& Sturdy, 2005; Horne \& Pearce, 2009b; Pearce, Graham, Good, Jones, \& McGregor, 2006); and in still others, such learning was potentiated by the landmark (Graham, Good, McGregor, \& Pearce, 2006; Pearce et al., 2006). One problem with interpreting these findings is that the experiments involved a variety of landmarks and, as we 
have just seen, a variety of arena shapes. It is thus difficult to identify the critical factors that determine when and how a landmark will influence spatial learning based on geometric cues. One factor that is beginning to be elucidated is gender (see Rodriguez, Torres, Mackintosh, \& Chamizo, 2010), but that does not explain why the various effects described above were shown solely with male subjects. One method for approaching this problem would be to use a variety of experimental manipulations with the same set of stimuli. By studying spatial learning in this systematic way, it should be possible to identify the conditions under which a landmark will augment, disrupt, or have no impact on learning about the location of a hidden goal with reference to geometric cues. With this rationale in mind, the present experiments can be viewed as a progression from a study by Horne and Pearce (2010).

The purpose of the previous study by Horne and Pearce (2010) was to determine whether a phenomenon known as superconditioning (see, e.g., Rescorla, 1971) could be demonstrated using a spatial task. An experimental group of rats was trained to escape from a rectangular pool of water by swimming to one of two escape platforms located just below the surface in diagonally opposite corners (A+ trials, where A represents the geometric cues unique to the correct corners). This training was continued in a subsequent phase, but a landmark, X, which had previously signalled the absence of a platform, was located close to each platform ( $\mathrm{AX}+$ trials). A control group was treated in the same way, except that the landmark was novel at the outset of the $\mathrm{AX}+$ trials. Thus, when it was introduced for the $\mathrm{AX}+$ trials, $\mathrm{X}$ would have inhibitory properties in the experimental group and no associative properties in the control group. During a test trial in the absence of $\mathrm{X}$ and the platforms, the experimental group spent significantly more time in the two correct corners than did the control group. Horne and Pearce (2010) explained this outcome by referring to theories of learning that assume that the increment in associative strength to a stimulus on a conditioning trial is determined by the discrepancy between the overall associative strengths of all of the stimuli present on a trial and the asymptote for conditioning set by the reinforcer, $\lambda$ (e.g., Pearce, 1994; Rescorla \& Wagner, 1972). The presence of the inhibitory $X$ at the outset of the $\mathrm{AX}+$ trials with the experimental group would ensure that the combined associative strength of the cues in a correct corner was less than $\lambda$, and thus provide further opportunity for A to gain associative strength. On the other hand, A would be expected to gain less associative strength in the control group, because the neutral associative strength of $\mathrm{X}$ would ensure that the overall associative strength of the cues in the correct corner was close to $\lambda$.

The experiment by Horne and Pearce (2010) took place in a rectangular pool with grey walls and landmarks that were either black or white laminated A4 cards pasted to the walls in the corners containing the platforms. The implication of the experiment, therefore, was that spatial learning based on these cues was governed by principles similar to those embodied in contemporary theories of learning (e.g., Pearce, 1994; Rescorla \& Wagner, 1972). The purpose of Experiment 1 was to test this conclusion in the simplest possible way by using an overshadowing design. Two groups of rats were trained to find one of two submerged platforms that were located in diagonally opposite corners of the same grey rectangle that had been used by Horne and Pearce (2010). For an experimental group, a landmark was always in each of the two corners containing the platform. The landmarks were the same as in the study by Horne and Pearce (2010). If spatial learning based on these cues were governed by the principles of associative learning theory, the presence of the landmarks should overshadow learning about the position of the platforms relative to the rectangular pool. To test this prediction, a control group was trained in a manner similar to that for the experimental group, except that on half of the trials the landmarks were in the two correct corners- those containing the platforms - and on the remaining trials they were situated in the two incorrect corners-those without the platforms. The landmarks were not expected to overshadow learning based on the geometric cues for this group. In order to determine the effectiveness of overshadowing, a test trial took place in the rectangular pool without the landmarks and the platforms. If the experimental group spent less time than the control group searching in the correct corners of the pool, it would indicate that overshadowing had been successful.

As was previously mentioned, the proposed experiment was not the first to investigate whether a landmark would overshadow spatial learning based on the shape of the environment. The findings from earlier studies, however, had been conflicting. Given these contrasting findings and the diverse ways in which they had been obtained, it was not at all clear whether overshadowing would be found in Experiment 1.

In fact, overshadowing was observed, but only partially. To adhere as closely as possible to the stimuli used by Horne and Pearce (2010), half of the rats in each group received a black card attached to the wall of the arena as the landmark, and for the remaining rats a white card served this purpose. The colours of the landmarks produced surprisingly different outcomes, and the experiment therefore concluded with a test trial to compare the control acquired by white and black landmarks in the two experimental groups. This trial took place in a square arena, with the appropriate landmark in one of the corners. Experiments $2 \mathrm{a}$ and $2 \mathrm{~b}$ were conducted in order to confirm the reliability of the effects revealed by Experiment 1 . The 
purpose of Experiment 3 was to examine the influence of geometric cues on learning about the black and white landmarks.

\section{Experiment 1}

\section{Method}

Subjects A group of 20 experimentally naive, male, hooded Lister rats (Rattus norvegicus), obtained from Harlan Olac (Bicester, Oxfordshire, England) and weighing between 250 and $300 \mathrm{~g}$ at the start of the experiment, was used. The rats were housed in white plastic cages with secured metal grid lids and maintained on a 12:12-h light:dark cycle, with lights on at 0700 . Subjects were housed in pairs and had continuous access to food and water in their home cages.

Apparatus A white, circular pool measuring $2 \mathrm{~m}$ in diameter and $0.6 \mathrm{~m}$ deep was used. The pool was mounted on a platform $0.6 \mathrm{~m}$ from the floor in the middle of the room $(4 \mathrm{~m} \times 4 \mathrm{~m} \times 2.3 \mathrm{~m})$. The pool was filled with water to a depth of $27 \mathrm{~cm}$ and was maintained at a temperature of $25^{\circ} \mathrm{C}\left( \pm 2^{\circ} \mathrm{C}\right)$. To make the water opaque, $0.5 \mathrm{~L}$ of white opacifer E308 (Roehm and Haas, U.K., Dewsbury) was used. The water was changed daily.

A white circular ceiling, measuring $2 \mathrm{~m}$ in diameter, was suspended $1.75 \mathrm{~m}$ above the floor of the pool. In the centre of the ceiling was a hole measuring $30 \mathrm{~cm}$ in diameter in which a video camera with a wide-angled lens was situated. The lens of the camera was $25 \mathrm{~cm}$ above the hole and was connected to a video monitor and computer equipment in an adjacent room. During tests, the rats' movements were analyzed using Watermaze software (Morris \& Spooner, 1990). The pool was illuminated by eight $45-\mathrm{W}$ lights that were located in the circular ceiling above the pool. The lights were $22.5 \mathrm{~cm}$ in diameter and were equidistant from each other in a $1.6-\mathrm{m}$ diameter circle whose centre was coincident with that of the circular ceiling. Two platforms, each measuring $10 \mathrm{~cm}$ in diameter and mounted on a column, were used during all training trials. The surface of each platform had a series of concentric ridges. For all trials, the base of the column rested on the bottom of the pool, and the platform surface was $2 \mathrm{~cm}$ below the surface of the water. A white curtain was drawn around the pool during all training and test trials. The curtain, which was attached to the edge of the circular ceiling, was $1.5 \mathrm{~m}$ high and fell $25 \mathrm{~cm}$ below the edge of the pool. Two black and two white landmarks were used in this study. Both landmarks consisted of two panels $(21 \mathrm{~cm} \times 29.7 \mathrm{~cm})$ that could be attached (via surface tension) to the walls, forming a corner in such a way that the longer edges of each panel made contact in the corner of the rectangle. The panels were composed of laminated A4 cards and were situated such that the bottom edge of each panel was $2 \mathrm{~cm}$ above the surface of the water.

Throughout the experiment, rats were trained in a rectangular-shaped pool constructed from two long grey Perspex boards ( $1.8 \mathrm{~m}$ long, $0.59 \mathrm{~m}$ high, and $2 \mathrm{~mm}$ thick) and two short grey Perspex boards $(0.9 \mathrm{~m}$ long, $0.59 \mathrm{~m}$ high, and $2 \mathrm{~mm}$ thick). Each board was placed vertically in the pool and suspended by bars that extended over the edge of the pool. For the landmark test, a square-shaped pool was also used. Each wall of the square was $1.41 \mathrm{~m}$ long, $0.59 \mathrm{~m}$ high, and $2 \mathrm{~mm}$ thick. The square was constructed from the same material and was the same colour as the rectangle.

Procedure Rats completed one session of four training trials each day. For each session, they were carried into a room adjacent to the test room in groups of 5 in a light-tight box. They remained in this box between trials. Each rat was carried from the box to the pool and was released facing the centre of a wall. The release point varied across trials, with each wall being used once in a given session. During a trial, the rat was required to swim to one of two submerged platforms. Each trial lasted a maximum of $60 \mathrm{~s}$. If the rat did not find the platform within $60 \mathrm{~s}$, the experimenter guided it to the closest platform. After climbing on the platform, the rat remained there for $20 \mathrm{~s}$ before being lifted from the pool, dried, and returned to its holding container. The intertrial interval for each rat was approximately $5 \mathrm{~min}$. Between trials, the experimenter rotated the arena $90^{\circ}, 180^{\circ}$, or $270^{\circ}$ clockwise. Four possible orientations were used (north, south, east, or west). The orientation of the arena across trials varied randomly, with the only stipulation being that each orientation was used once for any given session.

The rats were randomly assigned to two groups in equal numbers $(n=10)$. The experimental and control groups received 14 sessions of training with platforms located $25 \mathrm{~cm}$ from two of the corners in the rectangle, on an imaginary line that bisected the particular corner. For all of the rats, the platforms were located in the two corners in which the short wall was to the left of the long wall. Where the two groups differed was in the location of the landmarks. For the experimental group, the two landmarks - each comprising two panels - were attached to the walls forming the corners where the platforms were located. The locations of the landmarks for the control group were in the same corners as the platforms for half of the trials, and in the opposite corners for the other half of the trials. For half of the rats in each group, the colour of the panels was black, and for the remaining rats, the panels were white. 
The first three trials of Session 12 were conducted in the same manner as previous trials. The fourth trial consisted of a geometry test. For this test, the platforms and landmarks were absent from the pool. Rats were released from the centre of the rectangular pool and allowed to swim for $60 \mathrm{~s}$ before being removed from the pool. Following the test trial, a further, retraining session occurred. The fourth trial of Session 14 was a landmark test conducted in a squareshaped pool without a platform, but with a landmark in one corner of the square. Rats were released from the centre of the square and allowed to swim for $60 \mathrm{~s}$.

Throughout the experiment, except for the test trials, a record was taken of whether, after being released, a rat first entered one of the correct corners of the pool-the corners containing the platforms. A rat was deemed to have entered any of the four corners if its snout crossed a notional circular line with a radius of $40 \mathrm{~cm}$ and with its centre at the point where the walls creating the corner met. For ease of exposition, the term correct choice will be used to refer to those occasions on which a rat entered one of the correct corners before any of the other corners. For the purpose of analysing the results from the test trials in the rectangle and the square, circular search zones were used. Each search zone had a diameter of $30 \mathrm{~cm}$, with its centre positioned $25 \mathrm{~cm}$ from a corner on a line that bisected the corner. The percentages of time spent in the correct zones (where the platforms had been located during training trials) and the incorrect zones (the remaining two corners) of the rectangular pool were analysed. For the test trial in the square, the percentage of time spent in the correct zone (the zone near the landmark) and an average of the time spent in the remaining three, incorrect zones was used. A Type I error rate of .05 was adopted for all reported statistical comparisons.

\section{Results and discussion}

A striking feature of the two test trials was that the performance of the subjects from the experimental group trained with the white landmarks was different from the performance of those trained with the black landmarks. Accordingly, the results for all stages of the experiment are shown separately for subjects trained with the different landmarks.

The left-hand panel of Fig. 1 shows the mean escape latencies for the experimental and control groups throughout the training stage of the experiment. Both groups, irrespective of the colour of the landmark, became faster at finding one of the platforms as training progressed. By the end of training, there was no difference between the groups. A $2 \times 2$ Group $\times$ Colour ANOVA based on the individual mean latencies combined across Sessions 10, 11, and 12 revealed no significant main effects and no significant interaction, $F_{\mathrm{s}}(1,16)<2.78$.

The right-hand panel of Fig. 1 shows the mean percentages of correct choices across the 14 sessions of training for the various groups. The experimental subjects, irrespective of the colour of the landmark, made correct choices on virtually every trial from the outset. This strong preference for the correct corner at the beginning of training presumably reflects an unconditioned tendency to approach the landmark. The control rats started off at approximately chance and made fewer errors as training progressed. By the end of training, both the experimental and control groups were performing similarly. A Kruskal-Wallis test was conducted using individual mean percentages of correct choices combined across Sessions 10, 11, and 12. There was not a statistically significant difference between the groups, $H(3)=1.32$.

The left-hand panel of Fig. 2 shows the results from the geometry test in terms of the mean amount of time spent by each group in the correct and incorrect test zones of the rectangle. Overshadowing was revealed by the subjects trained with the white landmarks, as the control group spent a greater proportion of time in the correct zones than did the experimental group. The opposite of overshadowingpotentiation - was found with the subjects trained with the black landmarks, because now the experimental group spent a greater proportion of time in the correct zones than did the control group. A $2 \times 2 \times 2$ Group x Colour x Zone
Fig. 1 Mean $( \pm S E M)$ escape latencies (left-hand panel) and mean percentages of correct choices (right-hand panel) for the experimental and control groups of Experiment 1. B and $\mathrm{W}$ indicate black and white, the colours of the landmarks each group received during training
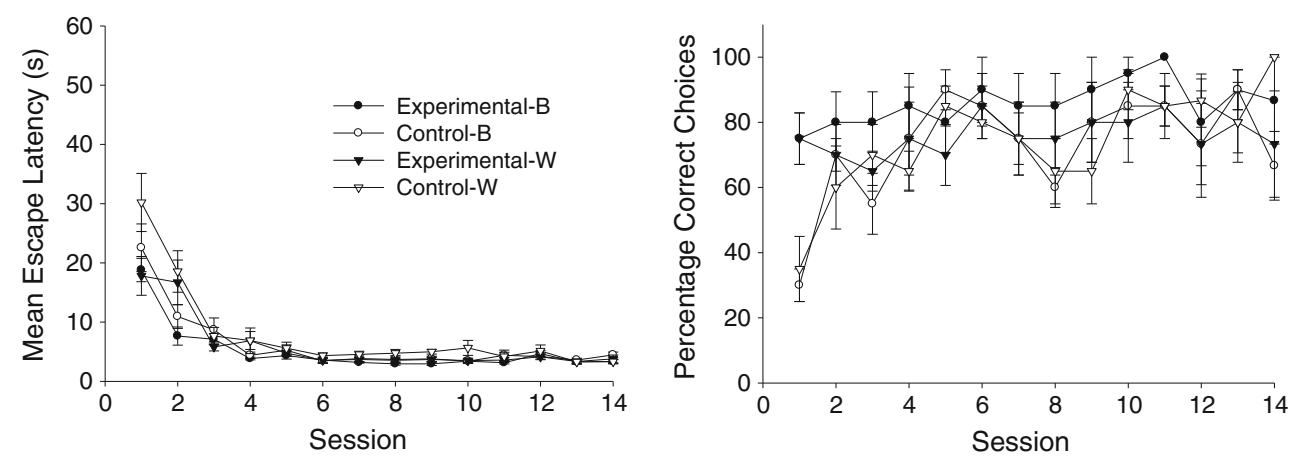
Fig. 2 Mean $( \pm S E M)$ percentage of time spent in the correct and incorrect zones during the geometry (left-hand panel) and landmark (right-hand panel) tests for the experimental and control groups of Experiment 1. $\mathrm{B}$ and $\mathrm{W}$ indicate black and white, the colours of the landmarks each group received during training
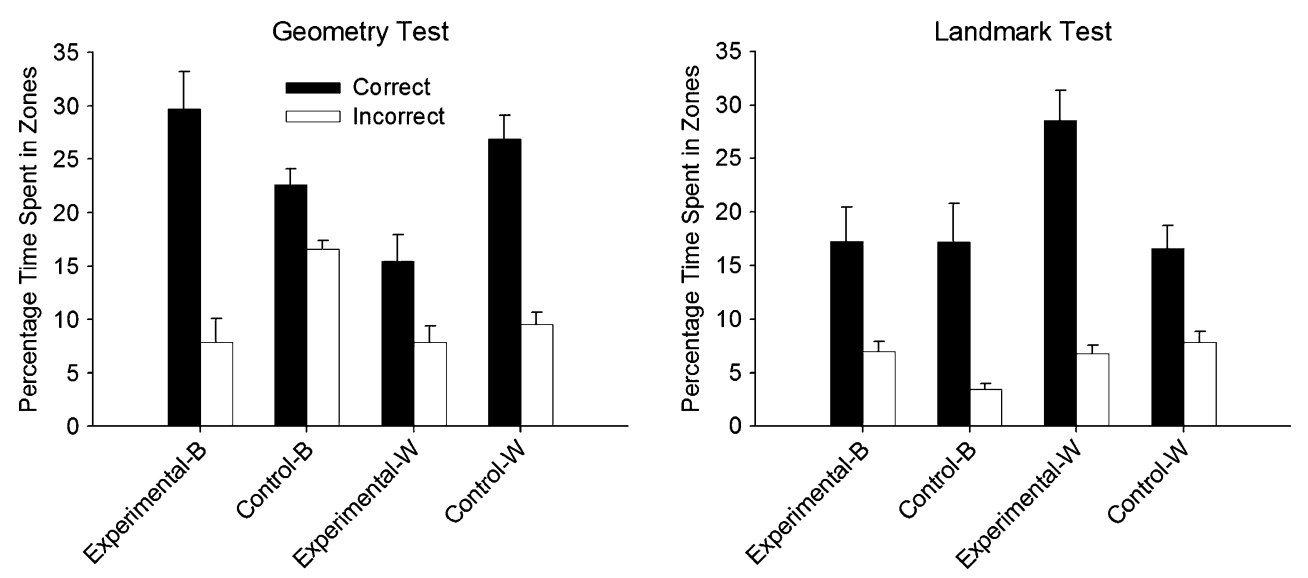

ANOVA was conducted and revealed significant main effects of group, $F(1,16)=7.68$, colour, $F(1,16)=10.33$, and zone, $F(1,16)=69.30$. There was also a significant Group $\mathrm{x}$ Colour interaction, $F(1,16)=4.76$, and a significant Group x Colour x Zone interaction, $F(1,16)=16.35$. The Group $x$ Zone and Colour x Zone interactions were not significant, $F_{\mathrm{s}}(1,16)<1$. A simple effects analysis on the three-way interaction revealed a significant Group x Zone interaction, $F(1,16)=12.59$, for the two groups trained with the black landmarks, and the experimental group spent more time in the correct zone than did the control group, $F(1,32)=6.05$. For the groups trained with the white landmarks, there was also a significant Group $\mathrm{x}$ Zone interaction, $F(1,16)=4.71$; however, unlike the groups trained with the black landmarks, the experimental group spent less time in the correct zone than did the control group, $F(1,32)=15.28$.

The right-hand panel of Fig. 2 shows the percentages of time spent in the correct and incorrect search zones of the square pool for the landmark test. The experimental rats that were trained and tested with the white landmarks spent considerably more time in the corner containing the landmark than did the experimental rats trained with the black landmarks. A similar difference was not evident for the rats in the control group, whose performance was similar to that of the rats from the experimental group trained with the black landmarks. A $2 \times 2 \times 2$ Group $\mathrm{x}$ Colour $x$ Zone ANOVA revealed a significant effect of zone, $F(1,16)=61.27$, and a Group x Zone interaction, $F(1,16)=5.43$. The three-way Group $\mathrm{x}$ Colour $\mathrm{x}$ Zone interaction was also significant, $F(1,16)=5.56$. All other main effects and interactions were not significant, $F_{\mathrm{S}}(1,16)<3.57$. A simple effects analysis on the three-way interaction revealed that each group showed a marked preference for the correct over the incorrect zones, $F_{\mathrm{S}}(1,16)>6.32$. In support of the foregoing observations, however, subjects belonging to the experimental group that were trained with white landmarks spent significantly more time near the landmark during the test trial than did either the rats in experimental group trained with the black landmarks, $F(1,32)=13.45$, or the control group trained with the white landmarks, $F(1,32)=15.08$. The two control groups spent approximately the same proportions of time in the correct zone, $F<1$.

The results from the experiment imply that when a hidden goal is located in the corner of a grey rectangular pool, a landmark in the same corner will overshadow learning based on the geometric cues when the landmark is white, and will augment such learning when the landmark is black. The results from the rats trained with white landmarks are in keeping with predictions from theories that assume that changes in associative strength are governed by a global error correction rule - that is, by a rule that takes account of the associative strengths of all of the cues that are present on a trial (e.g., Pearce 1994; Rescorla \& Wagner, 1972). In contrast, the results from the rats trained with the black landmarks stand in direct opposition to this rule. It is worth noting that during the test trial with the landmark, the experimental group trained with the white landmarks spent more time searching in the landmark's vicinity than did the group trained with the black landmarks. The implication of this finding is that the salience of the white landmarks was greater than that of the black landmarks, at least when presented against a grey background.

The theoretical implications of the present results are pursued further in the General Discussion, but first it is important to assess their reliability, for several reasons. First of all, as noted in the introduction to this experiment, demonstrations of overshadowing of geometric cues by a landmark are relatively rare. It is thus important to confirm that the results from the experimental and control groups trained with the white landmark are robust. This need is particularly pressing because there were only 5 rats in each of these subgroups. Similarly, demonstrations of potentiation of geometric cues by a landmark are also rare, and 
there is a need to confirm that the results from the rats trained with a black landmark are also reliable. Experiments $2 \mathrm{a}$ and $2 \mathrm{~b}$ were therefore conducted in an attempt to replicate the different results that were obtained with the black and white landmarks in Experiment 1, but with a greater number of rats. For Experiment 2a, an experimental and a control group were trained in the same way as their namesakes in the previous experiment, except that the landmarks were black for every subject.

\section{Experiment 2a}

\section{Method}

Subjects and apparatus The 20 naive male rats in this experiment were from the same stock, of similar weight, and housed in the same manner as those in Experiment 1. Ten of these rats were assigned at random to each of the two groups at the start of the experiment. The apparatus was identical to that of the previous experiment, except that the square-shaped pool was not used.

Procedure There were 12 sessions of training. The experimental and control groups were trained identically to their namesakes in Experiment 1, with two exceptions. First, only the black landmarks were used. Second, half of the animals received the platforms in the corners where the long wall was to the right of the short wall, and the other half received the platforms in the corners where the long wall was to the left of the short wall. The fourth trial of Session 12 was a geometry test conducted in the same manner as in Experiment 1. A landmark test was not included in this experiment.

\section{Results and discussion}

The left-hand panel of Fig. 3 shows the mean escape latencies for the experimental and control groups. Both groups were slow to find one of the platforms at the beginning of training but quickly became faster as training progressed. By the end of training, both groups were finding the platform in approximately the same amount of time. A $t$ test conducted with the individual mean latencies combined across the final three sessions revealed no significant differences, $t(18)=0.31$.

The right-hand panel of Fig. 3 shows the percentages of correct choices for each of the 12 training sessions. The experimental group again showed a preference for the correct corner, even at the beginning of training, while the control group started off making approximately 50\% correct choices upon being released into the pool. The performance of both groups improved as training progressed, but even at the end of this stage the performance of the experimental group was superior to that of the control group. A Mann-Whitney test based on individual mean percentages of correct choices combined across the final 3 sessions revealed a significant difference between the groups, $U(10,10)=23.5$.

Figure 4 depicts the group mean percentages of time spent in the correct and incorrect zones for the geometry test. In keeping with the results from Experiment 1, the experimental group spent a greater proportion of time in the correct zone than did the control group. A $2 \times 2$ Group $x$ Zone ANOVA revealed a significant main effect of zone, $F(1,18)=44.64$, and a significant Group $\mathrm{x}$ Zone interaction, $F(1,18)=7.83$. The group effect was not significant, $F(1,18)<1$. A simple effects analysis on the interaction confirmed the conclusions drawn from Experiment 1: The experimental group spent more time in the correct zone than did the control group, $F(1,36)=4.94$. In addition, both groups spent significantly more time in the correct than in the incorrect search zone, $F_{\mathrm{s}}(1,18)>7.53$.

In keeping with the results from Experiment 1, the black landmarks in the experimental group potentiated learning about the geometric cues, relative to the control group. The next experiment examined whether the opposite effect might be found when the landmarks were white rather than black.
Fig. 3 Mean ( \pm SEM) escape latencies (left-hand panel) and mean percentages of correct choices (right-hand panel) for the experimental and control groups of Experiment 2a
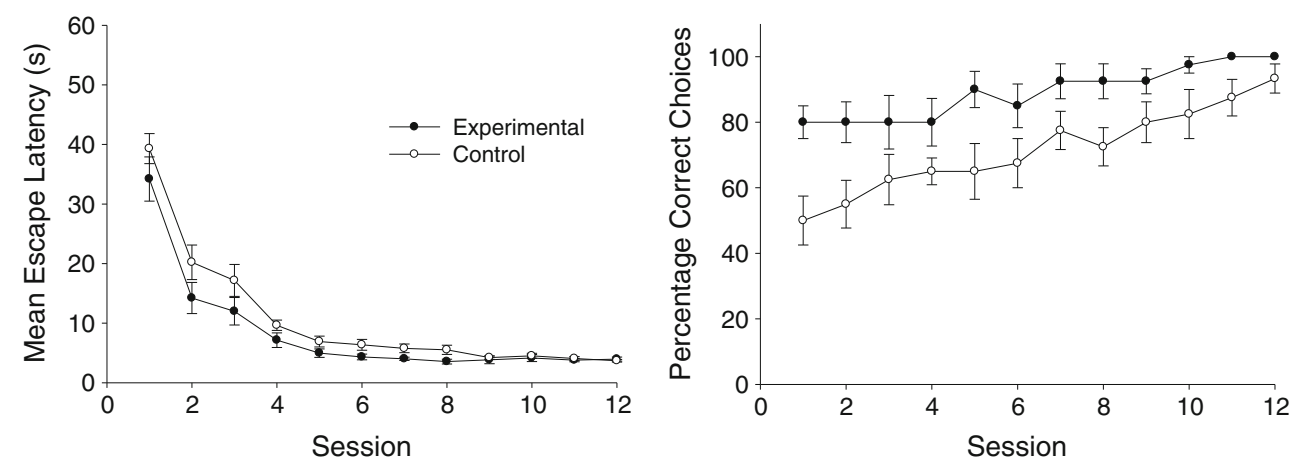


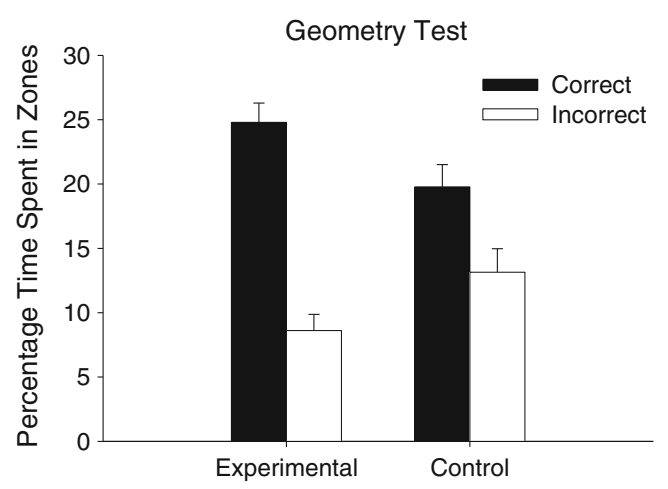

Fig. 4 Mean $(+S E M)$ percentages of time spent in the correct and incorrect zones during the geometry test for the experimental and control groups of Experiment 2a

\section{Experiment 2b}

The experiment was initially conducted with 10 rats in each of the two groups.

Although the test trial revealed that the control group spent more time than the experimental group searching in the correct corners of the pool, this difference was not significant. Accordingly, the experiment was repeated identically with a further 20 rats, and the results from both repetitions were then combined.

\section{Method}

Subjects, apparatus, and procedure The 40 male rats were from the same stock, of similar weight, and housed in the same manner as those in Experiment 1. Ten rats were assigned at random to each of the two groups at the start of each repetition of the experiment. The apparatus and procedure were identical to those of Experiment 2a, except that the landmarks were white rather than black.

\section{Results and discussion}

The left-hand panel of Fig. 5 shows the mean escape latencies for the two groups combined across the two repetitions of the experiment. The mean escape latencies for both groups became progressively shorter with continued training. A $t$ test was conducted with the individual mean latencies combined across the last three sessions. Contrary to appearance, the control group was significantly slower than the experimental group at finding the platform at the end of this stage, $t(38)=5.11$.

The right-hand panel of Fig. 5 shows the mean percentages of correct choices for the two groups across the 12 sessions of the experiment. The performance of both groups was similar at first, but by the end of training the percentage of trials on which a correct choice was made was significantly larger for the experimental than for the control group. A Mann-Whitney test, using individual mean percentages of correct choices combined across the last three sessions, revealed a significant difference between the groups, $U(20,20)=49.5$.

Figure 6 shows the group mean percentages of time in the correct and incorrect search zones during the test trial in the rectangular pool. The experimental group spent less time searching in the correct zones than did the control group. A $2 \times 2$ Group $x$ Zone ANOVA revealed significant main effects of group, $F(1,38)=7.32$, and zone, $F(1,38)=86.50$. Although the Group $x$ Zone interaction just failed to reach significance, $F(1,38)=3.75, p=.06$, the percentage of time spent in the correct search zones was significantly greater for the control than for the experimental group, $t(38)=2.50$. Thus, in keeping with Experiment 1, the presence of white landmarks in the corners of the rectangular pool that contained a platform overshadowed, rather than potentiated, spatial learning based on the geometric cues in the experimental group.

\section{Experiment 3}

One implication of the foregoing experiments is that the white landmark was in competition with the cues provided by the shape of the environment for the control it acquired over spatial behaviour. Learning about these different types of stimuli might therefore have been governed by a rule
Fig. 5 Mean ( $\pm S E M)$ escape latencies (left-hand panel) and mean percentages of correct choices (right-hand panel) for the experimental and control groups of Experiment $2 b$
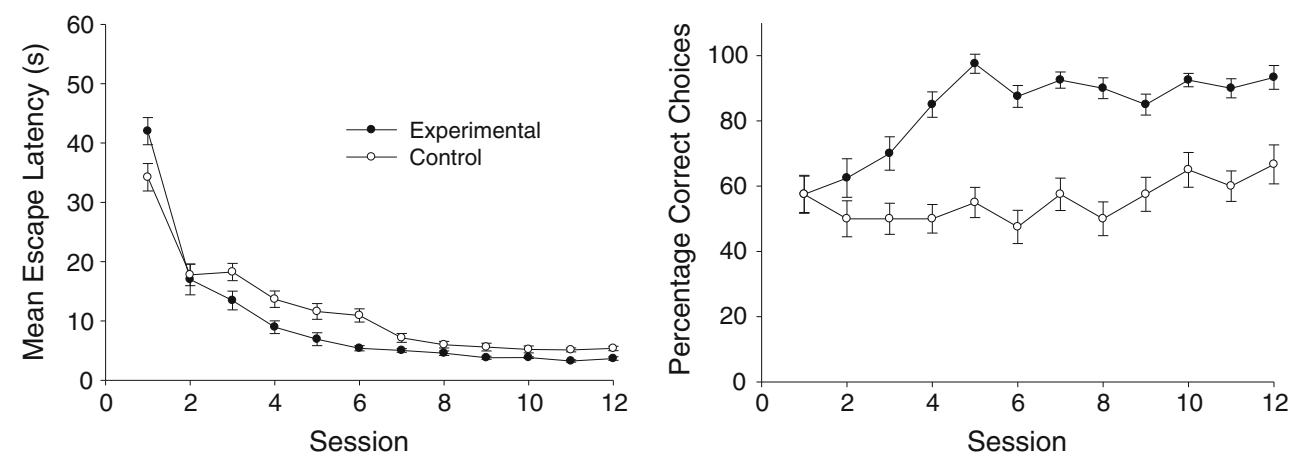


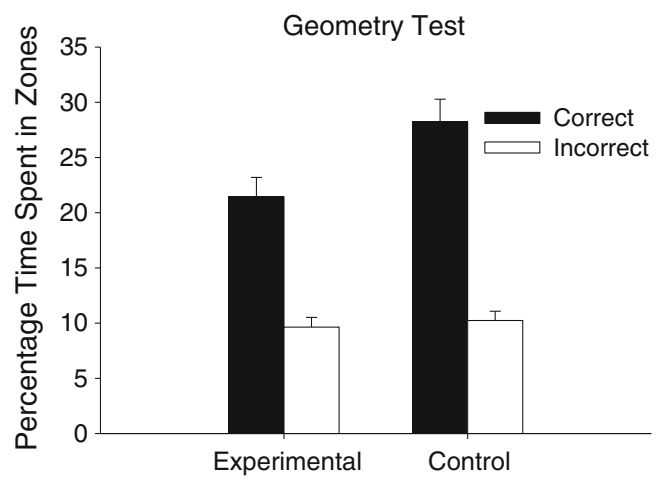

Fig. 6 Mean $(+S E M)$ percentages of time spent in the correct and incorrect zones during the geometry test for the experimental and control groups of Experiment $2 b$

similar to that believed to govern Pavlovian conditioning (see, e.g., Pearce, 1994; Rescorla \& Wagner, 1972). According to these theories, overshadowing should be reciprocal, so not only should the white landmark overshadow spatial leaning based on the geometric cues, but the geometric cues should also overshadow the white landmark. The main purpose of Experiment 3 was to test this prediction.

During the training stage of the experiment, two groups of rats were required to find one of two platforms located in diagonally opposite corners of a rectangle. For the experimental-W group, the platforms were always located in the same corners, and a white landmark was also located in each of these corners. For the control-W group, however, the platforms were placed in one pair of diagonally opposite corners for half of the trials, and in the other corners for the remaining trials. A white landmark was always in each of the corners containing the platforms. Thus, the platforms could be found by referring only to the white landmark in the control-W group, whereas they could be found by reference to either the landmark or the shape of the pool in the experimental-W group. The experiment concluded with a test trial in a square pool with the white landmark in one of the corners. Evidence of overshadowing of the white landmark by the geometric cues would be revealed in the test trial if the control-W group spent more time than the
experimental-W group searching for a platform in the corners containing the landmarks.

Two additional groups were included in the experiment: experimental-B and control-B. They were treated in the same way as the groups just described, except that the colour of the landmark was black rather than white. The purpose of these groups was to determine the generality of the effects found with the two groups trained with the white landmarks. In view of the different effects found with black and white landmarks in the previous experiments, it was hard to make any firm predictions concerning the outcome for these additional groups.

\section{Method}

Subjects and apparatus Ten male rats were assigned at random to each of the four groups at the start of the experiment. They were from the same stock, of similar weight, and housed in the same manner as those in Experiment 1. The apparatus was identical to that of Experiment 1.

Procedure The experimental-B and experimental-W groups were trained in the same way as the experimental groups of Experiments $2 \mathrm{a}$ and $2 \mathrm{~b}$, respectively. The remaining two groups were trained to swim to one of two submerged platforms that were always situated near a landmark. For the control-B group, the landmarks were black, and for the control-W group, the landmarks were white. For two of the trials within a session, the landmarks and the platforms were located in one pair of diagonally opposite corners, and for the remaining trials, they were located in the other two corners. The experiment lasted for 12 sessions, with a landmark test conducted in a square pool in the same manner as in Experiment 1 on the fourth trial of the final session.

\section{Results and discussion}

The left-hand panel of Fig. 7 shows the mean escape latencies for the four groups across the 12 training sessions.
Fig. 7 Mean $( \pm S E M)$ escape latencies (left-hand panel) and mean percentages of correct choices (right-hand panel) for the experimental and control groups of Experiment 3. B and $\mathrm{W}$ indicate black and white, the colours of the landmarks each group received during training
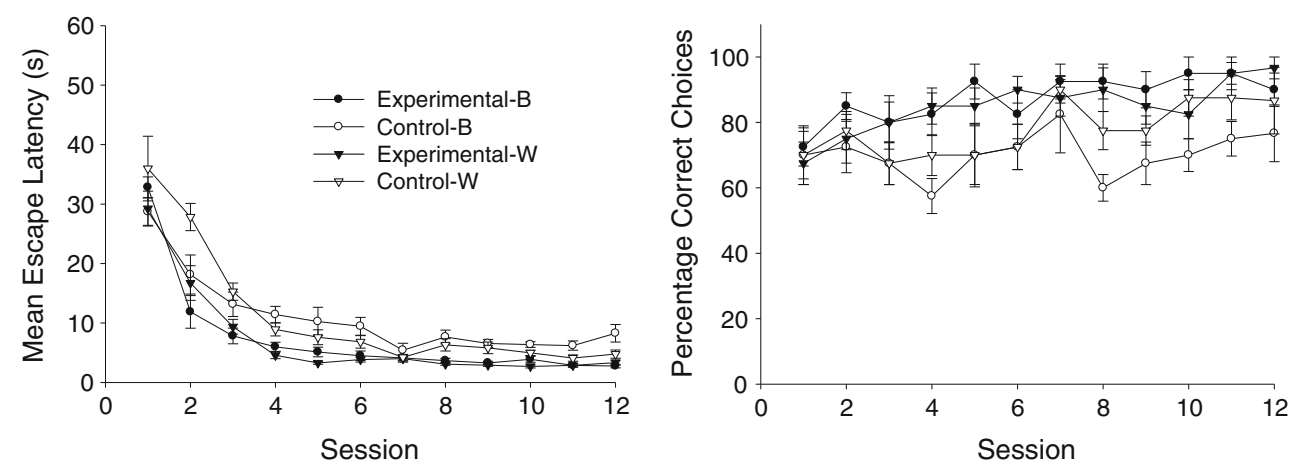
The performance by all of the groups improved as training progressed, but by the end of training the escape latencies for the control-B group were longer than those for the other groups. A $2 \times 2$ Group x Colour ANOVA was conducted on the individual mean escape latencies combined across the final three sessions. There were significant main effects of group, $F(1,36)=30.59$, and colour, $F(1,36)=6.76$, and a significant Group x Colour interaction, $F(1,36)=4.71$. A simple effects analysis conducted on the interaction revealed that the control-B and control-W groups took significantly more time to find one of the platforms than, respectively, the experimental-B and experimental-W groups, $F \mathrm{~S}(1,36)>5.64$. The escape latencies were shorter for the control-W group than for the control-B group, $F(1,36)=11.38$, but the difference between the two experimental groups was not significant, $F<1$.

The right-hand panel of Fig. 7 shows the group mean percentages of correct choices across the 12 sessions of training. Once again, by the end of training, the performance of the control-B group was inferior to that of the other three groups. A Kruskal-Wallis test was conducted on the individual mean percentages of correct choices combined across the final three sessions. The analysis revealed a difference among the groups, $H(3)=12.40$. A series of Mann-Whitney $U$ tests revealed that the control-B group made significantly fewer correct choices than did any of the other three groups, $U_{\mathrm{s}}(10,10)<19$, which did not differ, $U \mathrm{~s}(10,10)>38.4$.

Figure 8 shows the percentages of time spent searching in the correct and incorrect zones during the landmark test. The control-W group spent the greatest amount of time near the landmarks, the control-B spent the least amount of time near the landmarks, and the results for the experimental groups lay between these extremes. A $2 \times 2 \times 2$ Group $\mathrm{x}$ Colour $x$ Zone ANOVA revealed a significant effect of

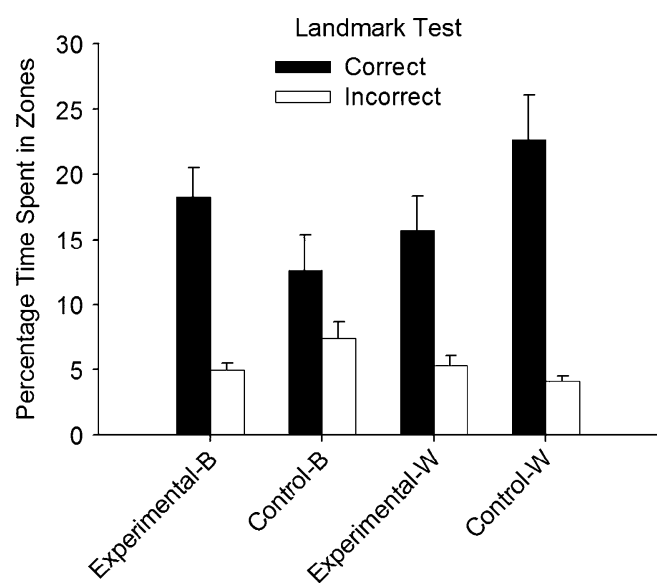

Fig. 8 Mean $(+S E M)$ percentages of time spent in the correct and incorrect zones during the landmark test for the experimental and control groups of Experiment 3. B and W indicate black and white, the colours of the landmarks each group received during training zone, $F(1,36)=50.79$, and a significant Group $\mathrm{x}$ Colour $\mathrm{x}$ Zone interaction, $F(1,36)=5.98$. All other main effects and interactions were not significant, $F_{\mathrm{s}}(1,36)<3.63$. A simple effects analysis of the three-way interaction revealed a significant Group $x$ Colour interaction for the time spent in the correct zones, $F(1,72)=9.58$, which indicates that the influence of the white landmark was different from that of the black landmark. Subsequent tests then revealed that the experimental-W group spent less time in the correct zone than did the control-W group, $F(1,72)=5.84$. Although numerically the experimental-B group spent more time in the correct zone than did the control-B group, this difference just fell short of the conventional level of significance, $F(1,72)=3.85, p=.05$. In addition, the control-W group spent more time in the correct zone during the test trial than did the control-B group, $F(1,72)=12.13$. The tests of simple effects further revealed that the control-B group failed to spend significantly more time in the correct than in the incorrect zone, $F(1,36)=2.44$, whereas each of the remaining groups spent more time in the correct than in the incorrect zone, $F \mathrm{~s}(1,36)>9.72$.

The results from the groups trained with the white landmarks show that the geometric cues overshadowed the white landmarks during the training with the experimental$\mathrm{W}$ group in the rectangle. Such an outcome is consistent with the suggestion that these different types of cues must compete for control over spatial behaviour in the manner envisaged by theories of associative learning (Pearce, 1994; Rescorla \& Wagner, 1972). As far as we are aware, only two other experiments have investigated whether cues provided by the boundary of the environment will overshadow spatial leaning based on a landmark. McGregor et al. (2009, Exp. 2) trained rats to find a submerged platform beneath a sphere in a distinctive corner of a triangularshaped swimming pool. In contrast to the present results, the geometric cues had no influence at all on control over searching for the platform by the landmark. In the other study, McGregor et al. (2009, Exp.3) found that the information provided by the black and white walls of a square arena was capable of overshadowing a spherical landmark that was suspended above a platform in one corner of the pool. Thus, the present experiment provides the first demonstration of spatial learning based on a landmark being overshadowed by geometric cues provided by the shape of the environment.

In keeping with Experiments 1 and 2, the present study again revealed that the pattern of results for the groups trained with white landmarks was very different from that for the groups trained with black landmarks. First of all, the results from the test trial with the control groups showed that the training in the rectangle resulted in the white landmark being more attractive than the black landmark. As the landmarks in these groups were the only 
cues that signalled where the platforms could be found during training, it is tempting to conclude that the salience of the white landmarks was greater than that of the black landmarks, and that conditioning was therefore more effective with the former than with the latter. The other difference between the effects of training with the black and white landmarks is that the geometric cues overshadowed spatial learning based on the white landmarks, and had the opposite effect with the black landmarks. The implications of this pattern of results will be considered in the General Discussion.

\section{General discussion}

Rats were required in each experiment to find one of two submerged platforms that were situated in diagonally opposite corners of a rectangular pool, and with a landmark beside each platform. When the landmarks were white, their presence overshadowed the control acquired by the geometric cues created by the shape of the pool, but when they were black, the landmarks enhanced or potentiated the influence of the geometric cues. Similarly, the geometric cues overshadowed learning about the landmarks when they were white, and had the opposite effect when the landmarks were black.

The results obtained with the white landmarks are consistent with the claim that learning in an environment with a distinctive shape is governed by a learning rule with a global error term (see, e.g., Pearce, 1994; Rescorla \& Wagner, 1972). Of course, the results obtained with the black landmark are opposite to those predicted by these theories. One way in which these contradictory results can be explained is to acknowledge that between-cue associations develop during spatial learning (Horne \& Pearce, 2009a; Rhodes, Creighton, Killcross, Good, \& Honey, 2009). When a rat finds a platform in one corner of a rectangular pool with a landmark near the platform, a number of associations might then develop. Associations are likely to develop between the landmark and the platform and between the geometric cues unique to the correct corner and the platform. Moreover, as one type of cue gains in strength, it may overshadow the other. It is also possible that the landmark and the geometric cues will enter into an association with each other. On a test trial without a landmark, therefore, the sight of the correct corner would activate the memory of the landmark, which in turn would activate a memory of the platform and encourage the rat to head for that corner. Thus, even though the correct corner might be weakly associated with the platform, through overshadowing, this effect might be more than compensated for through the indirect influence of the landmarkplatform association. Support for this analysis can be found in an experiment by Horne and Pearce (2009a) that demonstrated that associations do indeed develop between landmarks and geometric cues in a distinctively shaped environment.

What remains to be explained is why the white landmark should overshadow learning about the geometric cues, and the black landmark should potentiate it. A possible solution to this problem may rest with the results from the test trial with the two control groups of Experiment 3. The test revealed that the control group trained with the white landmarks spent more time searching near it than did the control group trained with the black landmarks, which suggests that ultimately the white landmarks were more attractive or salient than the black landmarks. In these circumstances, theories of learning predict that the overshadowing influence of the white landmark on the geometric cues would be greater than that of the black landmark. It would then be relatively easy for associations between the geometric cues and the black landmark to more than compensate for this modest effect of overshadowing and result in potentiation with the geometric cues. On the other hand, the between-cue associations involving the white landmark would have a larger deficit to overcome, and might therefore ameliorate but not reverse the effects of overshadowing by this landmark on the geometric cues.

Providing that the geometric cues and the white landmark were of similar salience, the foregoing explanation would also account for the overshadowing of the white landmark by the geometric cues that was seen in Experiment 3. To explain the potentiation with the black landmark by the geometric cues in that experiment, it would again have to be assumed that this landmark entered into a weak association with the platform. However, it might have entered into a strong association with the geometric cues, because of their spatial contiguity. If the geometric cues entered into a strong association with the goal, the strong association between the black landmark and the geometric cues could mediate a stronger response to the landmark than would be present for the group trained with only the black landmark as a cue for finding the platform.

An intriguing implication of the results from the groups trained with the black landmark is that potentiation between them and the geometric cues is reciprocal. Previous experiments have shown that training with a compound, $\mathrm{AB}$, will result in stimulus A potentiating stimulus $\mathrm{B}$, but whether the presence of B would also potentiate A was not investigated (see, e.g., Bouton, Dunlap, \& Swartzentruber, 1987; Durlach \& Rescorla, 1980; Graham et al., 2006). In fact, using taste aversion conditioning, Bouton et al. found that potentiation will occur only when the potentiated cue is of low salience and the potentiating cue is of intermediate salience. Given these constraints, it would not be possible for potentiation between two stimuli to be reciprocal. In 
view of the contradiction between this conclusion and the outcome of the present experiments with the black landmark, it is tempting to speculate that the principles governing potentiation may differ depending on the nature of the task or the cues that are used.

The original aim of the present experiments was to augment the results of Horne and Pearce (2010) by demonstrating overshadowing between the same landmarks and geometric cues that we had used for a successful demonstration of superconditioning. The results from the groups trained with the white landmarks fulfilled this aim, and thus lend support to the conclusion that spatial learning, at least with white landmarks and a rectangular pool, is governed by the principles of associative learning (e.g., Pearce, 1994; Rescorla \& Wagner, 1972). The results from the groups trained with the black landmarks, however, did not fulfil this aim, because they revealed potentiation rather than overshadowing. It is still possible that spatial learning based on black landmarks in a rectangular pool is governed by associative learning principles, but the effects of these principles may be modified by additional processes, such as between-cue associations, that can result in potentiation.

\section{References}

Bingman, V. P., Erichsen, J. T., Anderson, J. D., Good, M. A., \& Pearce, J. M. (2006). Spared feature-structure discrimination but diminished salience of environmental geometry in hippocampallesioned homing pigeons (Columba livia). Behavioral Neuroscience, 120, 835-841.

Bouton, M. E., Dunlap, C. M., \& Swartzentruber, D. (1987). Potentiation of taste by another taste during compound aversion learning. Animal Learning \& Behavior, 15, 433-438.

Cheng, K. (1986). A purely geometric module in the rats spatial representation. Cognition, 23, 149-178.

Durlach, P. J., \& Rescorla, R. S. (1980). Potentiation rather than overshadowing in odor-aversion learning: An analysis in terms of within-compound associations. Journal of Experimental Psychology: Animal Behavior Processes, 6, 175-187.

Gouteux, S., Thinus-Blanc, C., \& Vauclair, J. (2001). Rhesus monkeys use geometric and nongeometric information during a reorientation task. Journal of Experimental Psychology: General, 130, 505-519.

Graham, M., Good, M. A., McGregor, A., \& Pearce, J. M. (2006). Spatial learning based on the shape of the environment is influenced by properties of the objects forming the shape. Journal of Experimental Psychology: Animal Behavior Processes, 32, 44-59.

Gray, E. R., Bloomfield, L. L., Ferrey, A., Spetch, M. L., \& Sturdy, C. B. (2005). Spatial encoding in mountain chickadees: Features overshadow geometry. Biology Letters, 1, 314-317.

Hayward, A., Good, M. A., \& Pearce, J. M. (2004). Failure of a landmark to restrict spatial learning based on the shape of the environment. Quarterly Journal of Experimental Psychology, $57 B, 289-314$.

Hayward, A., McGregor, A., Good, M. A., \& Pearce, J. M. (2003). Absence of overshadowing and blocking between landmarks and the geometric cues provided by the shape of the test arena. Quarterly Journal of Experimental Psychology, 56B, 114-126.

Hermer, L., \& Spelke, E. S. (1996). Modularity and development: The case of spatial reorientation. Cognition, 61, 195-232.

Horne, M. R., \& Pearce, J. M. (2009a). Between-cue associations influence searching for a hidden goal in an environment with a distinctive shape. Journal of Experimental Psychology: Animal Behavior Processes, 35, 99-107.

Horne, M. R., \& Pearce, J. M. (2009b). A landmark blocks searching for a hidden platform in an environment with a distinctive shape after extended pretraining. Learning \& Behavior, 37, 167-178.

Horne, M. R., \& Pearce, J. M. (2010). Conditioned inhibition and superconditioning in an environment with a distinctive shape. Journal of Experimental Psychology: Animal Behavior Processes, 36, 381-394.

McGregor, A., Horne, M. R., Esber, G. R., \& Pearce, J. M. (2009). Absence of overshadowing between a landmark and geometric cues in a distinctively shaped environment: A test of Miller and Shettleworth (2007). Journal of Experimental Psychology: Animal Behavior Processes, 35, 357-370.

McGregor, A., Jones, P. M., Good, M. A., \& Pearce, J. M. (2006). Further evidence that rats rely on local rather than global spatial information to locate a hidden goal: Reply to Cheng and Gallistel (2005). Journal of Experimental Psychology: Animal Behavior Processes, 32, 314-321. doi:10.1037/0097-7403.32.3.314.

Morris, R. G. M., \& Spooner, R. I. W. (1990). Watermaze [Computer software]. Edinburgh, U.K.: Watermaze Software.

Pearce, J. M. (1994). Similarity and discrimination: A selective review and a connectionist model. Psychological Review, 101, 587-607.

Pearce, J. M., Good, M. A., Jones, P. M., \& McGregor, A. (2004). Transfer of spatial behavior between different environments: Implications for theories of spatial learning and for the role of the hippocampus in spatial learning. Journal of Experimental Psychology: Animal Behavior Processes, 30, 135-147.

Pearce, J. M., Graham, M., Good, M. A., Jones, P. M., \& McGregor, A. (2006). Potentiation, overshadowing, and blocking of spatial learning based on the shape of the environment. Journal of Experimental Psychology: Animal Behavior Processes, 32, 201214.

Pearce, J. M., Ward-Robinson, J., Good, M., Fussell, C., \& Aydin, A. (2001). Influence of a beacon on spatial learning based on the shape of the test environment. Journal of Experimental Psychology: Animal Behavior Processes, 27, 329-344.

Rescorla, R. A. (1971). Variation in the effectiveness of reinforcement and nonreinforcement following prior inhibitory conditioning. Learning and Motivation, 2, 113-123.

Rescorla, R. A., \& Wagner, A. R. (1972). A theory of Pavlovian conditioning: Variations in the effectiveness of reinforcement and nonreinforcement. In A. H. Black \& W. F. Prokasy (Eds.), Classical conditioning II: Current research and theory (pp. 6499). New York: Appleton-Century-Crofts.

Rhodes, S. E. V., Creighton, G., Killcross, A. S., Good, M., \& Honey, R. C. (2009). Integration of geometric with luminance information in the rat: Evidence from within-compound associations. Journal of Experimental Psychology: Animal Behavior Processes, 35, 92-98.

Rodriguez, C. A., Torres, A., Mackintosh, N. J., \& Chamizo, V. D. (2010). Sex differences in the strategies used by rats to solve a navigation task. Journal of Experimental Psychology: Animal Behavior Processes, 36, 395-401.

Sovrano, V. A., Bisazza, A., \& Vallortigara, G. (2003). Modularity as a fish (Xenotoca eiseni) views it: Conjoining geometric and nongeometric information for spatial reorientation. Journal of Experimental Psychology: Animal Behavior Processes, 29, 199210. 
Tommasi, L., \& Polli, C. (2004). Representation of two geometric features of the environment in the domestic chick (Gallus gallus). Animal Cognition, 7, 53-59.

Wall, P. L., Botly, L. C. P., Black, C. K., \& Shettleworth, S. J. (2004). The geometric module in the rat: Independence of shape and feature learning in a food finding task. Learning \& Behavior, 32, 289-298.

Wang, R. F., Hermer, L., \& Spelke, E. S. (1999). Mechanisms of reorientation and object localization by children: A comparison with rats. Behavioral Neuroscience, 113, 475-485.
Wystrach, A., \& Beugnon, G. (2009). Ants learn geometry and features. Current Biology, 19, 61-66.

\section{Author Note}

This research was supported by a grant from the Wellcome Trust. We thank Claire Pearson for her assistance in running Experiment 1. 